\title{
GENERALIZED DEGREE IN NORMED SPACES
}

\author{
Francisco Romero Ruiz del Portal
}

Abstract

We present a generalized degree theory for contimous maps $f$ : $(\bar{D}, \partial D) \rightarrow(E, E \backslash\{0\})$, where $E$ is a normed vectorial space, $D$ is an open subset of $B^{k} \times E$ such that $p_{1}(D)$ is bounded in $R^{k}$ and $f$ is a compact perturbation of the second projection $p_{2}: \mathbb{R}^{k} \times E \rightarrow E$.

\section{Introduction}

In 1934 J. Leray and S. Schauder extended the definition of the topological degree or Brouwer to compact perturbations of the identity in Banach spaces. They used finite-dimensional approximations of this compact functions ([D] and [LL]). If one consider the generalized degree theory presented in [G.M.V.] it is possible to extend it to the corresponding classes of maps in infinite-dimensional normed spaces.

Then the starting point is the degree theory given by Geba, Massabo and Vignoli in [G.M.V.] or the equivalent version, using framed cobordism, presented in $[R]$ and [R1]. We are going to extract briefly here, to do this paper as selfcontained as we can, the definitions and main results that we will need.

A $k$-notmally framed submanifold of $\mathbb{R}^{n+k}$ is a pair $\left(M^{k}, F\right)$ where $M^{k}$ is a compact submanifold of $\mathbb{R}^{n+k}$ and $F=\left\{u_{1}, \ldots, u_{n}\right\}$ is a normal frame for $M^{k}$. Two $k$-rormally framed manifolds of $\mathbb{R}^{n+k},\left(M_{0}, F_{0}\right)$ and $\left(M_{1}, F_{1}\right)$, are said to be homologous, if there exist a compact submanifold $M^{k+1}$ of $\mathbb{R}^{n+k} \times\{0,1]$ and a normal frame $G=\left\{v_{1}, v_{2}, \ldots, v_{\pi}\right\}$ for $M^{k+1}$ in $\mathbb{8}^{n+k} \times[0,1]$ such that:

1) $\partial M^{k+1}=M_{0} \times\{0\} \cup M_{1} \times\{1\}$.

2) $M^{k+1} \cap\left(\mathbb{R}^{n+k} \times\{t\}\right)=M_{0} \times\{t\}$ for every $t \in[0,1 / 3)$ and $M^{k+1} \cap$ $\left(\mathbb{B}^{n+k} \times\{t\}\right)=M_{1} \times\{t\}$ for every $t \in(2 / 3,1]$.

3) $\left.G\right|_{M_{0} \times\{0\}}=F_{0}$ and $\left.G\right|_{M_{2} \times\{1\}}=F_{1}$. 
The homology relation is an equivalence relation and let us denote $\vartheta_{c}^{k}\left(\mathbb{B}^{n+k}\right)$ as the set of the equivalence classes of $k$-normally framed submanifolds of $\mathbb{R}^{n+k}$.

L. Pontryagin showed $([\mathbf{P}])$ that there is a bijection $\Pi_{n !}^{k}: \Pi_{n+k}\left(S^{n}\right) \rightarrow$ $\vartheta_{c}^{k}\left(\mathbb{R}^{n+k}\right)$ defined by $\Pi_{n}^{k}([f])=\left[\left(\left(g \circ \varphi_{n+k}\right)^{-1}(p), F_{g^{\circ} \circ \varphi_{n+k}}\right)\right]$ where $\varphi_{n+k}$ : $\mathbb{R}^{n+k} \rightarrow S^{n+k} \backslash\left\{q^{\prime}\right\}$ is the inverse of the projection from the North Pole $q^{\prime}$ of $S^{n+k}, g: S^{n+k} \rightarrow S^{n}$ is a $C^{\infty}$ map homotopic to $f$, such that $p$ is a regular value of $g, q^{\prime} \notin g^{-1}(p)$ and $F_{g \circ \varphi_{n+k}}=\left\{u_{1}, u_{2}, \ldots, u_{n}\right\}$ is the normal frame for $\left(g \circ \varphi_{n+k}\right)^{-1}(p)$ in $\mathbb{R}^{n+k}$ such that $D\left(\varphi_{n+k}^{-1} \circ\right.$ $\left.g \circ \varphi_{n+k}\right)(x)\left(u_{j}(x)\right)=e_{j}$ for every $x \in\left(g \circ \varphi_{n+k}\right)^{-1}(p)$ and every $j \in$ $\{1,2, \ldots, n\}$ where $\left\{e_{1}, e_{2}, \ldots, e_{n}\right\}$ is the canonical basis of $\mathbb{R}^{n}$.

In $[R]$ and [R1] we follow an approximation process, similar to the diffcrential version of the classical degree theory of Brouwer, and therefore given a continuous map $f: \bar{\Omega} \subset \mathbb{R}^{n+k} \rightarrow \mathbb{R}^{n}$, defined in the closure of an bounded opcn subset $\Omega$ of $\mathbb{R}^{n}$, such that $0 \in \mathbb{R}^{n} \backslash f(\partial \Omega)$ we write the generalized degree of $f, d(f, \Omega)=d(g, \Omega)=\left(\Pi_{n}^{k}\right)^{-1}\left[\left(g^{-1}(0), F_{g}\right)\right] \in$ $\Pi_{n+k}\left(S^{n}\right)$, where $g: \bar{\Omega} \rightarrow \mathbb{R}^{n}$ is a $C^{\infty}$ map such that $\|f(x)-g(x)\|<$ $\operatorname{dist}(0, f(\partial \Omega))$ for every $x \in \bar{\Omega}$ and 0 is a regular value of $g$. One can prove that this definition and the definition of K. Geba, I. Massabó and A. Vignoli coincide.

This way of obtaining the gencralized degree present some advantages, for example we can point up that additivity and diffeomorphism invariance properties can be studied with more precision. Besides let us emphasizc that considering the group structure in $\vartheta_{c}^{k}\left(\mathbb{R}^{n+k}\right)$ we proved that additivity holds if $n \geq k+2$ and this is the best dimensional conditions for above property to hold. We also constructed examples, for arbitrary high dimensional cases, where additivity fails.

Now the extension of the generalized degree to proper maps is completely natural.

As we have mentioned additivity does not hold in general in finite-dimensional spaces. Howcver when one consider normed spaces of infinite dimension the generalized degree will satisfy additivity property without any restriction and the properties are analogous to the corresponding of the theory of Leray-Schauder (Corollary 9 and Proposition 10).

By making little changes to the constructions used to extend the degrec of Leray-Schauder to bigger classes of maps, for example $\gamma$-condensing perturbations, degree theory in locally convex spaces, etc., one also can extend this generalized degree to the corresponding classes of maps $([R])$.

Applications of this theory can be found in $[\mathbf{R}]$ and $[\mathbf{R 2}]$.

An equivariant generalized degree in Banach spaces from a different point of view has been given in [I.M.V.]. 


\section{Generalized degree for compact perturbations of the projection $p: \mathbb{R}^{k} \times E \rightarrow E$}

We start giving the basic notation and definitions

\section{Definition 1.}

Let $\left(E,\|\cdot\|_{E}\right)$ and $\left(F,\|\cdot\|_{F}\right)$ be two real normed spaces and $\Omega$ be a subset of $E$. A map $f: \Omega \rightarrow F$ is said to be compact if $f$ is continuous and $\overline{F(\Omega)}$ is a compact subset of $F$. We will write that $f$ is finitedimensional if $f(\Omega) \subset F_{n} \subset F$, where $F_{n}$ is a linear subspace of $F$ such that $\operatorname{dim} F_{n}=n$, for some $n \in \mathbb{N}$.

Denotc $\mathcal{K}(\Omega, F)=\{f: \Omega \rightarrow F: f$ is compact $\}$ and $\mathcal{F}(\Omega, F)=\{f:$ $\Omega \rightarrow F: f$ is compact and finite-dimensional $\}$.

The following lemmas are casy to prove

\section{Lemma 2.}

Let $\left(E,\|\cdot\|_{E}\right)$ and $\left(F,\|\cdot\|_{F}\right)$ be two normed spaces and $\Omega$ be a subset of $E$. Then for every $f \in \mathcal{K}(\Omega, F)$ and every $\varepsilon>0$ there exists $f_{\varepsilon} \in \mathcal{F}(\Omega, F)$ such that $\left\|f(x)-f_{\varepsilon}(x)\right\|<\varepsilon$ for all $x \in \Omega$.

\section{Lemma 3.}

Let $\left(E,\|\cdot\|_{E}\right)$ be a normed space and $\Omega$ be a closed subset of $\mathbb{R}^{k} \times E(k \in$ $\mathbb{N} \cup\{0\})$ such that $p_{1}(\Omega)$ is bounded $\mathbb{R}^{k}$ and let $f \in \mathcal{K}(\Omega, E)$. Then the map $g: \Omega \rightarrow E$ is proper.

$$
(\alpha, x) \stackrel{g}{\longmapsto} x-f(\alpha, x)
$$

Remark. Let us observe that if $p_{1}(\Omega)$ is unbounded it is casy to find examples where $g$ frils to be proper.

Let $k \in \mathbb{N} \cup\{0\}$. It is well known that the suspension homomorphism $\Sigma: \Pi_{n+k}\left(S^{n}\right) \rightarrow \Pi_{n+k+1}\left(S^{n+1}\right)$ is an isomorphism provided $n \geq k+2$. One can consider the directed set $\mathbb{N}$, with the usual order $\leq$, and the sequence of groups $\left\{\Pi_{k+n}\left(S^{n}\right)\right\}_{n \in \mathbb{N}}$. For $i \leq j$ there is a homomorphism $\Sigma_{i, j}: \Pi_{k+i}\left(S^{i}\right) \rightarrow \Pi_{k+j}\left(S^{j}\right)$ defined by $\Sigma_{i, j}=\Sigma_{j-1} \circ \Sigma_{j-2} \circ \cdots \circ \Sigma_{i}$. It. follows that $\Sigma_{i, i}=\mathrm{Id}$, and for every $i \leq j \leq 1$ one has $\Sigma_{i, 1}=\Sigma_{j, 1} \circ \Sigma_{i, j}$. Then, $\left\{\Pi_{k+n}\left(S^{n}\right), \Sigma_{i, j} i, j \in \mathbb{N}, \leq\right\}_{n \in \mathbb{N}}$ is direct system of groups. 
Denote by $\left(\Pi_{k}, \alpha_{i}\right), \alpha_{i}: \Pi_{k+i}\left(S^{i}\right) \rightarrow \Pi_{k}$, the direct limit of the above system. Therefore the diagram

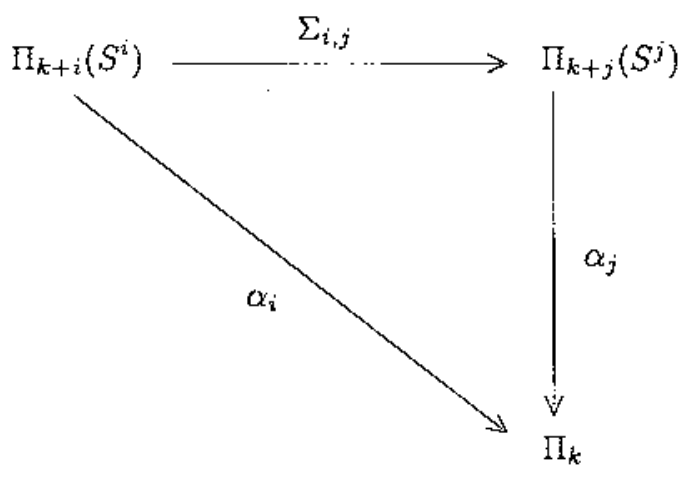

is commutative for cvery $i \leq j$.

Let us observe that $\Pi_{k}$ and $\Pi_{n+k}\left(S^{n}\right)$ are isomorphic if $n \geq k+2$.

The generalized degree that we are going to give will take values in $\Pi_{k}$.

Let $k \in \mathbb{N} \cup\{0\}, D$ be an open subset of $\mathbb{R}^{k} \times E$ such that $0 \in D$ and $p_{1}(D)$ is bounded in $\mathbb{B}^{k}$. Let $g: \bar{D} \rightarrow E$ be a map of the form $g(\alpha, x)=x-f(\alpha, x)$, where $f: \bar{D} \rightarrow E$ is compact and $0 \in E \backslash g(\partial D)$. It. follows that.

A) $r=\operatorname{dist}(0, g(\partial D))>0$.

Indeed, since $g$ is proper (Lemma 3 ), is a closed map. Hence $g(\partial D)$ is closed subset of $E$. Then therc exists $\varepsilon>0$ such that $B_{\varepsilon}(0) \cap g(\partial D)=\emptyset$ and $r \geq \varepsilon>0 .\left(B_{\varepsilon}(0)=\{x \in E: \operatorname{dist}(0 ; x)=\|x\|<\varepsilon\}\right.$.

B) If $r>0$ and $0<\varepsilon \leq r$, Lemma 2 implies that there exists a compact and finite-dimensional $T_{\varepsilon}$ such that $\left\|T_{\varepsilon}(z)-f(z)\right\|<\varepsilon$ for every $z \in \bar{D}$. Let us define $g_{\varepsilon}: \bar{D} \rightarrow E$ by $g(\alpha, x)=x-T_{\varepsilon}(\alpha, x)$ and $T_{\varepsilon}(\bar{D}) \subset L\left\{x_{1}, x_{2}, \ldots, x_{n}\right\}=V_{\varepsilon}$ (n-dimenisional subspace of $E$ ). Denote $D_{\varepsilon}=D \cap\left(\mathbb{R}^{k} \times V_{\varepsilon}\right)\left(D_{\varepsilon} \neq \emptyset\right.$, because $\left.0 \in D\right)$. Then $\bar{D}_{\varepsilon} \subset \bar{D} \cap\left(\mathbb{R}^{k} \times V_{\varepsilon}\right)$ and $g_{\varepsilon}\left(\bar{D}_{\varepsilon}\right) \subset V_{\varepsilon}$. Since $\mathbb{R}^{k} \times V_{\varepsilon}$ is closed $\mathbb{R}^{k} \times E$, the closure of $D_{\varepsilon}$ in $\mathbb{R}^{k} \times E$ and in $\mathbb{R}^{k} \times V_{E}$ coincide.

On the other hand, for each $(\alpha, x) \in \partial D_{\varepsilon}$,

$$
\begin{aligned}
\left\|, g_{\varepsilon}(\alpha, x)\right\|=\left\|x-T_{\varepsilon}(\alpha, x)+f(\alpha, x)-f(\alpha, x)\right\| \geq \\
\\
\geq\|x-f(\alpha, x)\|-\left\|f(\alpha, x)-T_{\varepsilon}(\alpha, x)\right\|>r-\varepsilon \geq 0 .
\end{aligned}
$$


Then the gencralized degree $d\left(\left.g_{\varepsilon}\right|_{\bar{D}_{\mathrm{s}}}, D_{\varepsilon}\right) \in \Pi_{n+k}\left(S^{n}\right)$ is well defined because $\left.g_{\varepsilon}\right|_{\bar{D}_{\varepsilon}}$ is a proper map ([G.M.V.] and [R]).

In order to continue we need the following lemma

\section{Lemma 4.}

Let $U$ be an open subset of $\mathbb{R}^{k} \times \mathbb{R}^{n} \equiv \mathbb{R}^{k+n}, 0 \in U$. Let $f: \bar{U} \rightarrow$ $\mathbb{R}^{n-1} \subset \mathbb{R}^{n}$ be a continuous map and $g: \bar{U} \rightarrow \mathbb{R}^{n}$ be the map defined by $g(\alpha, x)=x-f(\alpha, x)$. Suppose that $g$ is proper and that $0 \in \mathbb{R}^{n} \backslash g(\partial U)$. Then, $d(g, U)=\Sigma_{n-1}\left(d\left(\left.g\right|_{U \cap\left(\mathbb{B}^{k} \times \mathbb{R}^{n-i}\right)}, U \cap\left(\mathbb{R}^{k} \times \mathbb{R}^{n-1}\right)\right)\right)$.

\section{Proof:}

Using the homotopy invariance property there is no loss of generality in assuming that $f$ is a $C^{\infty}$ map and $0 \in($ r.v. $\left.)(g]_{v}\right)$ (the set of regular values of $\left.\left.g\right|_{J}\right)$.

It is clear that $g^{-1}(0)=\left(\left.g\right|_{\left(J \cap\left(\theta^{k} \times\left(\theta^{n-1}\right)\right.\right.}\right)^{-1}(0)$.

Let $(\alpha, x) \in g^{-1}(0)$. One can easily check that

$$
\operatorname{kcr} D g(\alpha, x)=\operatorname{ker} D\left(\left.g\right|_{U \cap\left(\mathbb{R}^{k} \times \mathbb{R}^{n}\right.}{ }_{1}\right)(\alpha, x) \subset \mathbb{R}^{k} \times \mathbb{R}^{n-1} .
$$

Then, if we denote by $N(\alpha, x)$ and $N^{\prime}(\alpha, x)$ the normal spaces to $g^{-1}(0)$ at $(\alpha, x)$ in $\mathbb{R}^{n+k}$ and $\mathbb{R}^{n+k-1}$ respectively, wo have that,

$$
N(\alpha, x)=N^{\prime}(\alpha, x) \oplus[(0,0, \ldots, 0,1)] .
$$

Let $\left.F_{\left(\left.g\right|_{L, \Gamma\left(\mathbb{R}^{*} \times{ }^{n-1}\right)}\right.}\right)=\left\{u_{1}, \ldots, u_{\pi t-1}\right\}$ be the normal frame for $g^{-1}(0)$ induced by $\left.g\right|_{U \cap\left(\mathbb{R}^{k} \times \mathbb{R}^{n \cdots 1}\right)}$.

Therefore $\left.D\left(\left.g\right|_{U \cap\left(\mathbb{R}^{*} \times \mathbb{R}^{n}\right.}\right)\right)\left((\alpha, x)\left(u_{j}(\alpha, x)\right)=c_{j}\right.$ for $\operatorname{cach} j \in\{1, \ldots, n-1\}$. Since $D g(\alpha, x)\left(e_{n+k}\right)=\left(\frac{-\partial f_{1}}{\partial x_{n}}, \ldots, \frac{-\partial f_{n}, 1}{\partial x_{n}}, 1\right)$ and $D g(\alpha, x)\left(u_{j}(\alpha, x)\right)=$ $e_{j}$, it, follows that the frame for $g^{-1}(0)$ induced by $g$ is $F_{g}=\left\{u_{1}, \ldots, u_{n_{2}-1}, u_{n_{2}}\right\}$ where $u_{n}(\alpha, x)=\frac{\partial f_{1}}{\partial x_{n}} u_{1}(\alpha, x)+\cdots+\frac{\partial f_{n-1}}{\partial \pi_{n}} u_{n-1}(\alpha, x)+e_{n+k}$.

Let $G_{1}: g^{-1}(0) \rightarrow G L_{+}\left(\mathbb{R}^{n}\right)$ be the $C^{\infty}$ rnap defired by

$$
G_{1}(\alpha, x)=\left(\begin{array}{cccc}
1 & \cdots & 0 & 0 \\
\vdots & & \vdots & \vdots \\
0 & \cdots & 1 & 0 \\
\frac{\partial f_{1}}{\partial x_{n}} & \cdots & \frac{\partial f_{t b-1}}{\partial x_{r_{l}}} & 1
\end{array}\right) .
$$

Then, the map $G: g^{-1}(0) \times I \rightarrow G L_{+}\left(\mathbb{R}^{n}\right)$ defined by

$$
G((\alpha, x): t)=\left(\begin{array}{ccccc}
1 & 0 & \cdots & 0 & 0 \\
\vdots & & & & \\
0 & 0 & \cdots & 1 & 0 \\
\frac{t \partial f_{1}}{\partial x_{n}} & \frac{t \partial f_{2}}{\partial x_{r l}} & \cdots & \frac{t \partial f_{n-1}}{\partial r_{r l}} & 1
\end{array}\right) .
$$


is $C^{\infty}, G_{0}=\mathrm{Id}$ and $G((\alpha, x), 1)=G_{1}(\alpha, x)$. Therefore $\left(g^{-1}(0) \times I, F\right)$ where $F((\alpha, x), t)=G((\alpha, x), t)\left(u_{1}, \ldots, u_{n-1}, e_{n+k}\right)$ achieves a homology between $\left(g^{-1}(0), F_{g}\right)$ and $\left(g^{-1}(0),\left\{u_{1}, \ldots, u_{n-1}, e_{n+k}\right\}\right)$. Then

$$
\begin{aligned}
& {\left[\left(g^{-1}(0), F_{g}\right)\right]=\left[\left(g^{-1}(0), \Sigma F_{\left(\left.g\right|_{\text {Uri }\left\{\mathbf{I}^{k} \times \boldsymbol{m}^{n} \cdot 1\right.}\right)}\right)\right]=} \\
& \left.=\Sigma\left(\left[\left(\left.g\right|_{U \cap\left(\mathbb{R}^{k} \times \mathbb{F}^{n-1}\right)}\right)^{-1}(0), F_{\left(\left.g\right|_{U \sim\left(\mathbb{R}^{k} \times \mathbb{R}^{n} \cdot 1\right.}\right)}\right)\right]\right) \text {. }
\end{aligned}
$$

(see $[\mathrm{P}])$.

Wc are now in a situation of define the generalized degrce when $D$ is an opcn subset of $\mathbb{B}^{k} \times E, 0 \in D, p_{1}(\bar{D})$ is bounded and the map $g:(\bar{D}, \partial D) \rightarrow(E, E \backslash\{0\})$ is defined by $g(\alpha, x)=x-f(\alpha, x)$, where $f: \bar{D} \rightarrow E$ is a compact map.

\section{Definition 5.}

Let $\varepsilon \leq r=\operatorname{dist}(0, g(\partial D))$ and $T_{\varepsilon}$ be a compact finite-dimensional approximation of $f$ such that $\left\|f(\alpha, x)-T_{\varepsilon}(\alpha, x)\right\|<\varepsilon$ for every $(\alpha, x) \in$ $\bar{D}$. We dofine $d(g, D)=\alpha_{n}\left(d\left(g_{\varepsilon}, D_{\varepsilon}\right)\right) \in \Pi_{k}$, where $g_{\varepsilon}(\alpha, x)=x-$ $T_{\varepsilon}(\alpha, x), D_{\varepsilon}=D \cap\left(\mathbb{B}^{k} \times V_{\varepsilon}\right)$ and $n=\operatorname{dirm} V_{\varepsilon} \quad\left(T_{\varepsilon}(\bar{D}) \subset V_{\varepsilon}\right)$.

\section{Lemma 6.}

The generalized degree, defined in Definition 4 does not depend on $\varepsilon, V_{\varepsilon}$ and $T_{\varepsilon}$.

\section{Proof:}

Let $0<\varepsilon \leq r$ and $0<\eta \leq r$. Let us consider $V_{p^{2}}=L\left\{V_{\varepsilon}, V_{\eta}\right\}$. Assurne that $\operatorname{dim} V_{k^{2}}=s, \operatorname{dim} V_{\varepsilon}=n$ and $\operatorname{dim} V_{\eta_{p}}=m$. Let $D_{\mu}=D \cap V_{k}$, then $T_{\varepsilon}(\bar{D}) \subset V_{\varepsilon} \subset V_{\mu}$ and $T_{\eta}(\bar{D}) \subset V_{\eta} \subset V_{1}$. Lemma 4 inplies that $d\left(g_{\varepsilon}, D_{\mu}\right)=\sum_{n, s}\left(d\left(g_{\varepsilon}, D_{\varepsilon}\right)\right)$ and $d\left(g_{\eta}, D_{\mu}\right)=\Sigma_{m, s}\left(d\left(g_{\eta}, D_{\mu}\right)\right)$.

On the other hand, the map $H: \bar{D}_{\mu} \times I \rightarrow V_{\mu}$, defined by $H(z, t)=$ $t g_{\varepsilon}(z)+(1-t) g_{\eta}(z)$, satisfies that $H\left(\left(\partial D_{\mu}\right) \times I\right) \subset V_{\mu} \backslash\{0\}$, then $d\left(g_{\varepsilon}, D_{\mu}\right)=$ $d\left(g_{n}, D_{i^{2}}\right)$. Consequently

$$
\begin{gathered}
\alpha_{n_{i}}\left(d\left(g_{\varepsilon}, D_{\varepsilon}\right)\right)=\left(\alpha_{s} \circ \Sigma_{\eta, s}\right)\left(d\left(g_{\varepsilon}, D_{\varepsilon}\right)\right)=\alpha_{s}\left(d\left(g_{\varepsilon}, D_{\mu}\right)\right)=\alpha_{s}\left(d\left(g_{\eta} . D_{\mu}\right)\right)= \\
=\left(\alpha_{s} \circ \Sigma_{\eta, s}\right)\left(d\left(g_{\eta}, D_{\eta}\right)\right)=\alpha_{m}\left(d\left(g_{\eta}, D_{\eta}\right)\right) .
\end{gathered}
$$

\section{Definition 7 (General case).}

Let $k \in \mathbb{N} \cup\{0\}, E$ be a normed space, $D$ be an open subset of $\mathbb{R}^{k} \times E$ such that $p_{1}(D)$ is bounded in $\mathbb{R}^{k}$. Let $g:(\bar{D}, \partial D) \rightarrow(E, E \backslash\{0\})$ be a map of the form $g(\alpha, x)=x-f(\alpha, x)=\left(p_{2}-f\right)(\alpha, x)$, where $f: \bar{D} \rightarrow E$ is a compact map and $p_{2}(\alpha, x)=x$. We define

$$
d(g, D)=d\left(g \circ \tau_{\left(\alpha_{0}, x_{0}\right)}, G_{\left(\alpha_{0}, x_{0}\right)}\right) \in \Pi_{k}
$$


where $\left(\alpha_{0}, x_{0}\right)$ is an arbitrary element of $D, G_{\left(\alpha_{0}, x_{0}\right)}=D-\left(\alpha_{0}, x_{0}\right)$, $\tau_{\left(\alpha_{0}, x_{0}\right)}: G_{\left(\alpha_{0}, x_{0}\right)} \rightarrow D$ is the map defined by $\tau_{\left(\alpha_{0}, x_{0}\right)}(\alpha, x)=(\alpha, x)+$ $\left(\alpha_{0}, x_{0}\right)$ and $d\left(g \circ \tau_{\left(\alpha_{0}, x_{0}\right)}, G_{\left(\alpha_{0}, x_{0}\right)}\right) \in \Pi_{k}$ is an in Definition $5((0,0) \in$ $G_{\left(\alpha_{0}, x_{0}\right)}$ and $\left(g \circ \tau_{\left(\alpha_{0}, x_{0}\right)}\right)(\alpha, x)=x-\left(f\left(\alpha+\alpha_{0}, x+x_{0}\right)-x_{0}\right)$ satisfies $\left.0 \notin\left(g \circ \tau_{\left(\alpha_{0}, x_{0}\right)}\right)\left(\partial G_{\left(\alpha_{0}, x_{0}\right)}\right)=g(\partial D)\right)$.

Remark. Obviously one has to justify that Definition 7 does not depend on the choice of $\left(\alpha_{0}, x_{0}\right)$.

Let $\left(\alpha_{0}, x_{0}\right)$ and $\left(\alpha_{1}, x_{1}\right)$ be two arbitrary elements of $D$.

Assume that $\varepsilon<r=\operatorname{dist}(0, g(\partial D))=\operatorname{dist}\left(0,\left(g \circ \tau_{\left(\alpha_{0}, x_{0}\right)}\right)\left(\partial G_{\left(\alpha_{0}, x_{0}\right)}\right)\right)=$ $\operatorname{dist}\left(0,\left(g \circ \tau_{\left(\alpha_{1}, x_{1}\right)}\right)\left(\partial G_{\left(\alpha_{1}, x_{1}\right)}\right)\right)$.

There exists a map $T_{\varepsilon} \in \mathcal{F}(\bar{D}, E)$ such that $\left\|T_{\varepsilon}(\alpha, x)-f(\alpha, x)\right\|<\varepsilon$ for every $(\alpha, x) \in \bar{D}$. Let. $V$ be a finite-dimensional subspace of $E$ such that $T_{\varepsilon}(\bar{D}) \subset V, V_{\varepsilon}=L\left\{V \cup\left\{x_{0}, x_{1}\right\}\right\}$ and $n=\operatorname{dim} V_{\varepsilon}$.

We consider the compact and finite-dimensional maps $T_{\varepsilon}^{0}: \bar{G}_{\left(\alpha_{0}, x_{0}\right)} \rightarrow$ $V_{\varepsilon}, T_{\varepsilon}^{1}: \bar{G}_{\left(\alpha_{1}, x_{1}\right)} \rightarrow V_{\varepsilon}$, defined by $T_{\varepsilon}^{0}(\alpha, x)=T_{\varepsilon}\left(\alpha+\alpha_{0}, x+x_{0}\right)-x_{0}$ and $T_{\varepsilon}^{1}(\alpha, x)=T_{\varepsilon}\left(\alpha+\alpha_{1}, x+x_{1}\right)-x_{1}$ respectively. Since $\| T_{\varepsilon}^{0}(\alpha, x)-$ $\left(f\left(\alpha+\alpha_{0}, x+x_{0}\right)-x_{0}\right) \|<\varepsilon$ for every $(\alpha, x) \in \bar{G}_{\left(\alpha_{0}, x_{0}\right)}$ and $\| T_{\varepsilon}^{1}(\alpha, x)-$ $\left(f\left(\alpha+\alpha_{1}, x+x_{1}\right)-x_{1}\right) \|<\varepsilon$ for every $(\alpha, x) \in \bar{G}_{\left(\alpha_{1}, x_{3}\right)}$, it follows that $d\left(g \circ \tau_{\left(\alpha_{0}, x_{0}\right)}, G_{\left(\alpha_{0}, x_{0}\right)}\right)=\alpha_{n}\left(d\left(g_{\varepsilon}^{0}, D_{\varepsilon}^{0}\right)\right)$ and $d\left(g \circ \tau_{\left(\alpha_{1}, x_{1}\right)}, G_{\left(\alpha_{1}, x_{1}\right)}\right)=$ $\alpha_{n}\left(d\left(g_{\varepsilon}^{1}, D_{\varepsilon}^{1}\right)\right)$, where $g_{\varepsilon}^{0}=p_{2}-T_{\varepsilon}^{0}, g_{\varepsilon}^{1}=p_{2}-T_{\varepsilon}^{1}, D_{\varepsilon}^{0}=G_{\left(\alpha_{0}, x_{0}\right)} \cap\left(\mathbb{R}^{k} \times\right.$ $\left.V_{\varepsilon}\right)$ and $D_{\varepsilon}^{1}=G_{\left(\alpha_{1}, x_{1}\right)} \cap\left(\mathbb{R}^{k} \times V_{\varepsilon}\right)$.

We have to check that $d\left(g_{\varepsilon}^{0}, D_{\varepsilon}^{0}\right)=d\left(g_{\varepsilon}^{1}, D_{\varepsilon}^{1}\right)$.

It is clear that $\varphi=\tau_{\left(-\alpha_{1},-x_{1}\right)} \circ \tau_{\left(\alpha_{x}, x_{0}\right)}=\tau_{\left(-\alpha_{1}+\alpha_{0},-x_{1}+x_{0}\right)}$ is a homeomorphism from $\overline{D_{\varepsilon}^{0}}$ ont $\delta \overline{D_{\varepsilon}^{1}}$ such that $g_{\varepsilon}^{1} \circ \varphi=g_{\varepsilon}^{0}$. Now, using that $\varphi_{n+k} \circ \varphi \circ \varphi_{r_{t}+k}^{-1}: \varphi_{n+k} \overline{\left(D_{\varepsilon}^{1}\right)}$ can be extended to a homeomorphism $h: S^{n+k} \rightarrow S^{n+k}$ of degree 1 , from I.1.11 of [R] (or [R1]) we have that $d\left(g_{\varepsilon}^{0}, D_{\varepsilon}^{0}\right)=d\left(g_{\varepsilon}^{1} \circ \varphi, D_{\varepsilon}^{0}\right)=d\left(g_{\varepsilon}^{1}, D_{\varepsilon}^{1}\right)$.

\section{Lemma 8.}

Let $D$ be an open subset of $\mathbb{R}^{k} \times E$ such that $p_{1}(\bar{D})$ is bounded in $\mathbb{R}^{k}$. Let $g: \vec{D} \rightarrow E$ be a map of the form $g(\alpha, x)=x-T(\alpha, x)$ where $T$ is a compact map, $0 \in E \backslash g(\partial D)$ and $r=\operatorname{dist}(0, g(\partial D))$. Let $S: \bar{D} \rightarrow E$ be another compact map such that $\|S(\alpha, x)-T(\alpha, x)\|<r / 2$ for every $(\alpha, x) \in \bar{D}$. Let us consider $h: \bar{D} \rightarrow E$ defined by $h(\alpha, x)=x-S(\alpha, x)$. It follows that $0 \in E \backslash h(\partial D)$ and $d(h, D)=d(g, D)$. 


\section{Proof:}

If $\left(\alpha_{0}, x_{0}\right) \in D$, for every $(\alpha, x) \in G_{\left(\alpha_{0}, x_{0}\right)}=D^{\prime}-\left(\alpha_{0}, x_{0}\right)$ one has that $\left\|S\left(\alpha+\alpha_{0}, x+x_{0}\right)-x_{0}-\left(T\left(\alpha+\alpha_{0}, x+x_{0}\right)-x_{0}\right)\right\|<r / 2$. Then there is no loss of generality in assuming that $0 \in D$.

1) Let $x \in \partial D$ then $\|h(\alpha, x)\|=\|h(\alpha, x)-g(\alpha, x)+g(\alpha, x)\| \geq$ $\|g(\alpha, x)\|-\|h(\alpha, x)-g(\alpha, x)\| r-r / 2>0$.

2) Let. $\varepsilon=r / 4, T_{\varepsilon}: \bar{D} \rightarrow E$ and $S_{\varepsilon}: \bar{D} \rightarrow E$ be the corresponding compact and finite-dimensional maps such that $\| T(\alpha, x)-$ $T_{\varepsilon}(\alpha, x) \|<r / 4$ and $\left\|S(\alpha, x)-S_{\varepsilon}(\alpha, x)\right\| r<4$ for every $(\alpha, x) \in$ $\bar{D}, T_{\varepsilon}(\bar{D}) \subset V_{\varepsilon}^{T}$ and $S_{\varepsilon}(\bar{D}) \subset V_{\varepsilon}^{S}$. Let $V=L\left\{V_{\varepsilon}^{T} \cup V_{\varepsilon}^{S}\right\}$ and $\operatorname{dim} V=n$. It is clear that $d(h, D)=\alpha_{n}\left(d\left(h_{\epsilon}, D_{v}\right)\right)$ and $d\left(g_{1} D\right)=\alpha_{n_{k}}\left(d\left(g_{\varepsilon}, D_{v}\right)\right)$ where $h_{\varepsilon}(\alpha, x)=x-S_{\varepsilon}(\alpha, x), g_{\varepsilon}(\alpha, x)=$ $x-T_{\varepsilon}(\alpha, x)$ and $D_{V}=D \cap\left(\mathbb{R}^{k} \times V\right)$.

Now we define the nnap $F: \bar{D}_{V} \times I \rightarrow V$ by $F((\alpha, x), t)=t h_{\varepsilon}(\alpha, x)+$ $(1-t)_{g_{\varepsilon}}(\alpha, x)$. It is straightforward that $\|F((\alpha, x), t)\|>0$ for all $(\alpha, x) \in$ $\partial D V \subset \partial D$ and $t \in I$.

Therctore $d\left(g_{\varepsilon}, D_{V}\right)=d\left(h_{\varepsilon}, D_{V}\right)$ and conscopuently $d(g, D)=d(h, D)$.

\section{Corollary 9 (Homotopy invariance property).}

Let $D$ be an open sulset of $\mathbb{R}^{k} \times E$, such thot $p_{1}(D)$ is bounded in $\mathbb{R}^{k}$. Let $H: \bar{D} \times I \rightarrow E$ be a compact map and $F: \bar{D} \times I \rightarrow E$ be defined by $F((\alpha, x), t)=x-H((\alpha, x), t)$. Assume that $0 \in E \backslash F(\partial D \times I)$, hence $d\left(F_{t}, D\right) \quad t \in[0,1]$ is constant.

\section{Proof:}

Since $H$ is compact Lemma 3 irmplies that $F: \bar{D} \times I \rightarrow E$ is a proper map. Then $F(\partial D \times I)$ and dist $(0, F(\partial D \times I))>0$. Consider the compact set $K=\overline{H(\bar{D} \times I)} \subset E$. Since $I d_{K}: K \rightarrow E$ is a compact map there is a compact and finite-dimensional map $T: K \rightarrow E$ such that $\| T(x)-$ $I d_{K}(x) \|<r / 2$ for every $x \in K$.

Let in define $G: \bar{D} \times I \rightarrow E$ by $G((\alpha, x), t)=z-(T \circ H)((\alpha, x), t)$ ard let $V$ be a subspace of $E$ such that $T(K) \subset V$ and dim $V=n$.

For each $y \in \bar{D} \quad\left\|H_{t}(y)-\left(T \circ H_{t}\right)(y)\right\|<r / 2$, then $d\left(F_{t}, D\right)=$ $d\left(G_{i}, D\right)$. On the other hand $d\left(G_{t}, D\right)=\alpha_{n}\left(d\left(G_{i}\right)_{\bar{D} \cap\left(\mathbb{B}^{k} \times V\right)}, D \cap\left(\mathbb{R}^{k} \times\right.\right.$ V)) has constant value for $t \in[0,1]$. Therefore $d\left(F_{l}, D\right)$ is constant $t \in[0,1]$. 


\section{Proposition 10.}

The generalized degree given in Definition 7 has also the follouing properties:

1) Solution. If $d(g, D) \neq 0$, then there exists $\left(\alpha_{0}, x_{0}\right) \in D$ such that $g\left(\alpha_{0}, x_{0}\right)=0$.

2) Excision. Let $U \subset D$ be an open such that $g(D \backslash U) \subset E \backslash\{0\}$. Then one has that $d\left(\left.g\right|_{\bar{U}}, U\right)=d(g, D)$.

3) Additinity. Let $U_{1}$ and $U_{2}$ be open subsets contained in $D$ such that $U_{1} \cap U_{2}=$ and $g\left(D \backslash\left(U_{1} \cup U_{2}\right)\right) \subset E \backslash\{0\}$. It follows that $d(g, D)=d\left(\left.g\right|_{\bar{U}_{1}}, U_{1}\right)+d\left(\left.g\right|_{\bar{U}_{2}}, U_{2}\right)$.

The proof is easy and we will ornit it.

Remark. It is interesting to point out that additivity property of above proposition implies that onc can construct a generalized degree, $d^{\prime}$, in linear finite-dimensional spaces (as a particular case of normed spaces) such that $d^{t}$ always satisfics additivity property. However in many caes we would lose an important, information because if $d(f, U) \in \Pi_{7 b+k}\left(S^{n}\right)$ is not trivial and $\Sigma(d(f, U))=0$, one would have that $d^{\prime}(f, U)=0$. Then using the solution property of the generalized degrce, $d$, presented in [G.M.V.] one deduce that there exists solutions of the equation $f(\alpha, x)=0$. This is not possible with $d^{\prime}$.

Acknowledgement. I wart to thank Professor E. Outcrelo for his assistance and for all of his valuable time that he dedicates to me while the thesis proparation.

\section{References}

[D] K. Deimuine, "Nonlinear Functional Analysis," Springer Verlag, 1985.

[G.M.V.] K. Gizisa, I. Massabó and A. Vignom, Generalized degree and bifurcation, Nonlinear Analysis and its Applications (1986), $55-73$.

[H] Monnis W. Hinsci, "Differential Topology," Springer Verlag, 1976.

[Hu] S. T. Hu, "Homotopy Theory," Acadernic Press, 1959.

[I.M.V.] J. IzE, I. MASSAibó and A. Vignofi, Degree theory for equivariant maps I, Trans. Amer. Math. Soc. 315 (1989), 433-510.

[LL] N.G. LıoY), "Degree Theory," Cambridge: University Press, 1978. 
[P] L.S. PONTRYaGix, Smooth manifolds and their applications in Homotopy Theory, Ameer. Math. Soc. Translats. 11 (1959), 1-114.

[R] F.R. RuIz DEL PORTAL, Teoría del grado topológico generalizado y aplicaciones, Tesis.

[R1] F.R. RUI2 DEL PORTAL, On the additivity property of the gencralized degree, Math. Joponica, to appear.

[R2] F.R. RuIz DEL PORTAL, Generalized complementing maps, Preprint.

Unidad Dorente de Maternatiks Aplicada

E.'T.S.I. de Montes

Lniversidad Politćcnica de Madrid

Cindad Lniversitaria

28010 Madrid

SPAIN

Rebut el 8 d'Abril de 1991 